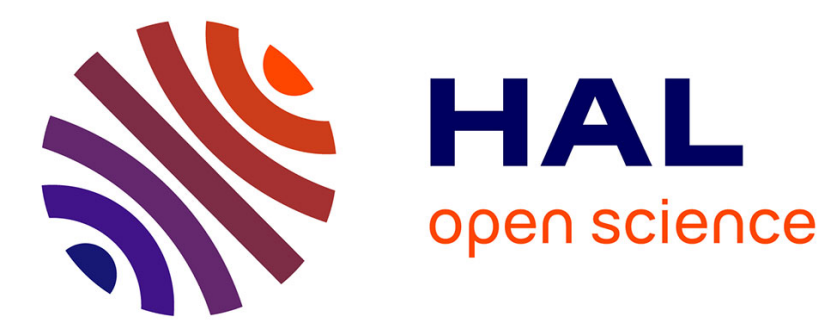

\title{
WeKeyInnovation, A Wiki Based on Crowdsourcing to Share Information about Innovation Support
}

\author{
Jérémie Faham, Nawel Takouachet, Jérémy Legardeur
}

\section{To cite this version:}

Jérémie Faham, Nawel Takouachet, Jérémy Legardeur. WeKeyInnovation, A Wiki Based on Crowdsourcing to Share Information about Innovation Support. IFIP International Conference on Advances in Production Management Systems (APMS), Sep 2014, Ajaccio, France. pp.289-297, 10.1007/9783-662-44739-0_36. hal-01069904

\section{HAL Id: hal-01069904 https://hal.science/hal-01069904}

Submitted on 27 Oct 2016

HAL is a multi-disciplinary open access archive for the deposit and dissemination of scientific research documents, whether they are published or not. The documents may come from teaching and research institutions in France or abroad, or from public or private research centers.
L'archive ouverte pluridisciplinaire HAL, est destinée au dépôt et à la diffusion de documents scientifiques de niveau recherche, publiés ou non, émanant des établissements d'enseignement et de recherche français ou étrangers, des laboratoires publics ou privés. 


\title{
WeKeylnnovation, a Wiki Based on Crowdsourcing to Share Information about Innovation Support
}

\author{
Jérémie Faham ${ }^{1}$, Nawel Takouachet ${ }^{1}$, and Jérémy Legardeur ${ }^{1,2}$ \\ ${ }^{1}$ ESTIA, F-64210 Bidart, France \\ $\{j$.faham, n.takouachet, j. legardeur\} destia.fr \\ ${ }^{2}$ IMS, UMR 5218, Talence, France
}

\begin{abstract}
One of the challenges to foster innovation is to understand the existing practices and the real needs to help companies when they initiate and develop innovative projects or new ideas. This paper addresses this question by proposing a new interactive and collaborative support based on the crowdsourcing approach. We propose a collaborative wiki platform WeKeylnnovation (WKI), which will be used and enriched progressively by companies, consulting and institutional. To identify specific companies' needs, we introduce how this guide has been developed promoting a collaborative environment to share and evaluate good practices, tools, software or theories about creativity and innovation. Furthermore, our WKI solution will allow building a dynamic observatory by collecting empirical and valuable ground data on their real practices and needs. The final purpose is to define most efficient policies and enhance the formulation of a real regional strategic plan toward an economical growth based on innovation.
\end{abstract}

Keywords: Innovation, Small and Medium Enterprises, Crowdsourcing, Interactive Platform, Open Innovation, Collaboration, Entrepreneurship.

\section{Introduction}

It is now a well-established fact that creativity and innovation stimulation is a key issue for company's development and competitiveness. This topic has been widely studied either in the social, economic and management sciences or in the engineering fields. Most of the times global analysis are based on questions and surveys that are push to companies in order to have a feedback on their needs and practices, aiming to identify their barriers and levers faced to innovation processes [14].These approaches are often promoted by different stakeholders (researchers, governmental institutions...) and lead to the publication of different documentation [13] that provides global information according to the situation of the country, the size and the type of the company, the field of activity... On the other hand, research-action based on empirical studies within companies also produces qualitative results about the deep understanding of sociotechnical and economical aspects of innovation processes [1]. However, classical methods (enquiries, surveys, soundings, diagnostics...) commonly used in Social and Human Sciences and Engineering Sciences are limited regarding to methodological (lake of reliability, insufficient answers rates...) and operational aspects (waste of time, duplications, lake of interest for the organizations investigated...). 
In this paper, we attempt to overcome these limitations by proposing WeKeyInnovation (WKI), an open interactive and collaborative support based on the crowdsourcing approach [2]. Indeed, it can be used and enriched progressively by any individual or organization interested in innovation processes.

Our open and collaborative platform is designed according to two main objectives:

- The first is to give an online access to information related to existing methods, tools, software, funding, consulting... that can be used by companies to manage and support their creativity and innovation processes. This information is progressively proposed and updated by all the stakeholders. This raw information is reviewed and classified by advanced users or experts in the field of creativity and innovation management that can be composed of researchers, consulting, associations... It has to offer an intuitive front office providing a free access to qualitative information reviewed by experts.

- The second objective is to provide a dynamic observatory to allow some specific statistics and elicitations concerning the practices, the needs, the levers and the barriers for companies faced to innovation challenges. This collected ground data is helpful to define new innovation policies and politics enabling then the implementation of better designed strategic plans toward an economical growth based on innovation.

This paper is structured as follows. In Section 2 we suggest 10 key points we identified throughout the existing literature about creativity and innovation processes within enterprises. In section 3 we show how our WKI features answer those 10 key points we underlined. In section 4 we present the technical prerequisites that our WKI must integrate to satisfy our objectives and insure a successful implementation. In section 5 we discuss the future implications of this work before concluding with some directions for future work.

\section{Background Literature Review}

We reviewed existing literature regarding levers, confines, difficulties and limits of innovation processes in companies [3,4,5,6,7,8,9,10,11,12,13,14]. We identified and selected ten significant key points that may reduce the existing gap between the real needs and the usual tools or measures commonly used to answer enterprises desires to implement creativity and innovation processes, as following:

1. Consider innovation as a collective and iterative process.

2. Open and crowd innovation driven by the promotion of partnerships and a free access to knowledge to enhance the creation of new skills and solutions which didn't exist until now.

3. Introduce both educational and entrepreneurship values based on a broad creativity mindset to develop "innovation culture" outside and within organizations. This implies a wide demystification and democratization of "innovation perception" by the education of individuals and especially SMEs owners, showing them that innovation is surely not only a technologic high added value process restricted to big enterprises and always requiring wide ID capabilities. 
4. Increase interdisciplinary projects and diversified culture within organizations by building real "cross-disciplinary innovative teams".

5. Create the conditions to promote a smart collaboration outside, within and between organizations, aiming to enhance an economic sustainable growth based on the knowledge.

6. Reinforce collaboration with a better networking and circulation of information to stimulate exchanges and links between all innovation stakeholders and elaborate a real "innovative ecosystem".

7. Design efficient strategic innovation policies, based on real enterprise's needs, weaknesses or strengths. This involves relying on a qualitative and qualified raw data collection.

8. Pay attention to "societal innovation" by focusing on advances for the society and highlighting the new collaborative innovation development models to satisfy societal challenges implications.

9. Insure a central role of public authorities and regions in strategic innovation policies definition and implementation to ensure good spillovers on the wider national and thus international economic society.

10. Introduce new tools that catalyze the needs, the skills and centralize the best know-how. This should allow creating an open network and a common language by arranging the contributions of different fields. Those new tools have to support both human resources and technologic advances bringing together new sustainable development paradigm and numeric revolution challenges to design new sustainable development models and growing strategies for SMEs.

Those ten key points lead us to design our WeKeylnnovation to reduce the gap existing between company's needs, practices and classical supports traditionally used. The main purpose is to help defining better policies to foster innovation.

\section{WeKeyInnovation Features}

The purpose is to develop a collaborative platform opening creativity and innovation processes to all individuals and companies with a special focus on SMEs. In this section, in order to illustrate and answer the key points we presented in the previous section, we will introduce our main proposal: the design of an interactive and collaborative opened platform (1) (2) (4) (10) designed to support SMEs in their operational daily tasks and enhance the creativity and innovation processes they would like to start, improve or implement. The idea is to develop a way toward a real "innovation culture" based on a broad creativity mindset within the WKI community we created (3) (10).

One of the main key point we assert to answer implementing WKI is to better understand the practices of all the organizations by collecting raw data without having to resort to traditional methods. Hence, our solution attempts to help designing efficient and adequate strategic innovation policies starting from real enterprise's needs, customs, weaknesses or strengths automatically collected in real time on our WKI (7) (9). $V i a$ this automatic treatment of information raised from the observed interactions, the followed trajectories and inputs provided or filled on the web platform by the WKI users, we expect a better understanding of real enterprises and individuals confines or 
needs to design then better policies and satisfy societal challenges implications (8) (9) (10). Already in the elaboration stage of the process, the WeKeylnnovation is comprehended as an interactive platform dedicated to support enterprises in both their operational needs and innovative processes, based on serious play and crowdsourcing concepts (1) (2) (4), usable online by enterprises, individuals, institutional and consulting organizations via a pioneering web portal (6) (10). We pay a particular attention to SMEs as they constitute the core of the European but also international economic and productive network. Regarding to this requirement, our platform will create a smart supportive environment (5) enabling an efficient networking to stimulate exchanges and links between all innovation participants (6) (10).

\section{Technical Prerequisites}

As a first stage of WeKeyInnovation design, we intend to insure a set of technical features to develop an efficient framework enable to further a qualitative, fast and intuitive enrichment of the platform.

\subsection{Scalable and Flexible Arborescent Architecture}

To allow a smart collaborative interaction between users and stimulate a progressive auto-enrichment, we design an open architecture enabling to process, rank, comment or rate users contributions. But to insure a successful implementation we first need to integrate a pre-organization of data collected during our "state of the art" process. A first classification has been proposed according to the literature (innovation guides, creativity methods, norms, web sites links, skills reports, solutions, existing software platforms, free open data bases...). On the other hand, contributions proposals are organized in a tree categories structure composed by information about the whole supporting and consulting measures, tools or helps existing to support innovation at large, such as: Methods, Tools, Expertise, Diagnostics, Suggestions, Events, Testimonies, Good practices... The arborescence is flexible enough to be later progressively and collectively enriched or expanded by all the users of the community. Indeed, according to his/her profile, each user can investigate, add, qualify, evaluate or approve contributions. He can also classify or modify contributions categories. The figure (Fig.1) shows the example of the possible reviewer actions on WKI.

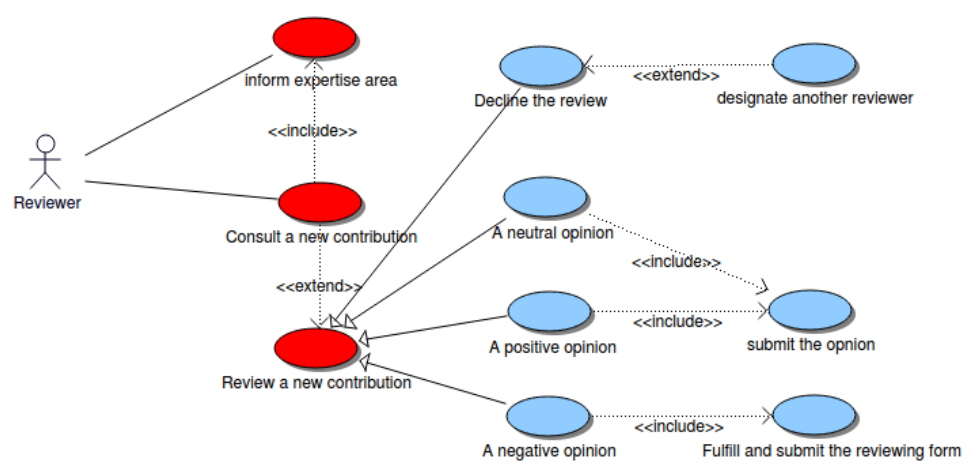

Fig. 1. UML use case diagram of the reviewer role 
Those contributions are filled in the WKI by users through predefined templates we already designed to ensure an easy flow of information (Fig. 2).
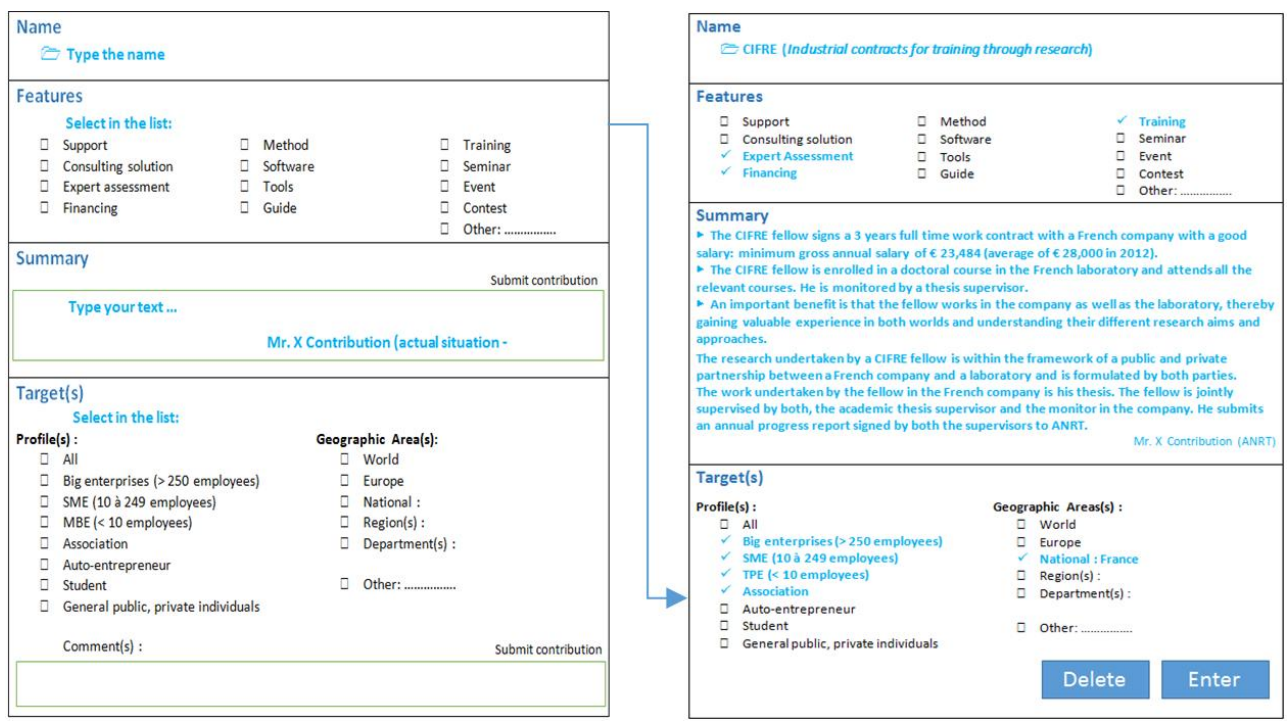

Fig. 2. An example of the WKI template

\subsection{An Intuitive Front Office Interface}

Another challenge is to provide a fast and efficient use compatible with all kinds of computer supports. WKI must ensure pertinent answers according to the user's operational needs, while leading them to implement progressively innovation processes. This feature brings us to think on a smart design which promotes an intuitive browsing easily understandable "by and for the whole stakeholders", especially for our main specific target: SMEs owners. In addition of classical research functions based on keywords, we integrate an advanced research browser offering a smart intuitive and precise need formulation to satisfy each individual's expectations. The figure 3 illustrates an example of a WKI advanced research sequence.

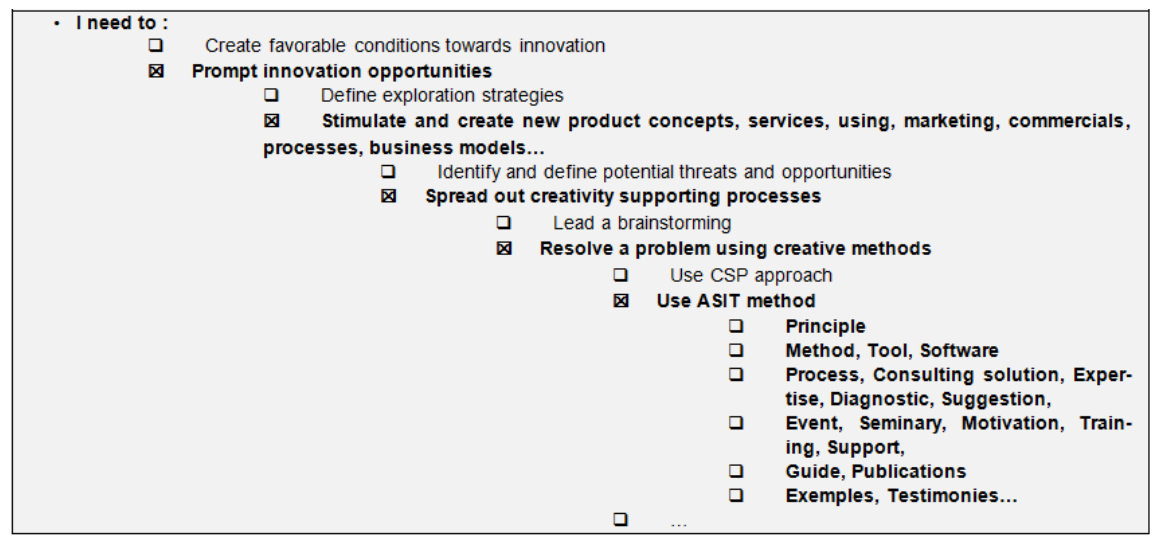

Fig. 3. An advanced research WKI sequence example 


\subsection{A Collaborative and Interactive online resource SMEs-Orientated}

This aspect will enable us to build a dynamic observatory of organizations practices relying on an empirical and real-time data collection of real individual's and enterprises strengths or expectations. SMEs constitute one of the main stakeholders among the new ongoing innovation dynamics. Admittedly they are fragile but globally their economic and human strength presents a strong potential able to change the general situation. However, according to the report "Pour une nouvelle vision de l'innovation" [6] only $15 \%$ of French SMEs are considered as innovative enterprises (14th rank in European Union).

Nevertheless, SMEs capacities to learn, product, transfer knowledge and values as their reactivity, agility and their adaptive potential are impressive. Indeed, SMEs must be investigated with strong attention if we plan to enhance innovation processes a large scale and highlight those particular enterprises potential in both entrepreneur and academic worlds. Constituting one of the main targets of WKI, we reviewed a consistent state of art focusing on creativity and innovation processes within SMEs $[3,4][7]$. Relying on these findings, we design the WKI contents towards SMEs characteristic obligations to release their strong innovative potential. Our WKI features take into account real SMEs needs and obstacles being as well a smart support to communicate around their strengths and good practices.

\subsection{Securing Environment}

Our wish for an open and free-access to useful knowledge for the society at whole shouldn't mask the importance we attach to confidentiality and data securing, being both essential requirements for our tool. Indeed, the maximal reduction of the risk is a necessary prerequisite to achieve the creation of a secure environment. This must absolutely be seriously considered, especially when dealing with economic organizations and SMEs to ensure a free flow of information exchanges and the development of innovation processes in a safe climate based on confidence within the new community.

Regarding to this topic, we design so a controlled access for users by making them log in to WKI using their professional e-mail address or their professional social networks account. A beta test of WKI is already planned within a selected community. The objective is to guarantee its proper functioning in operating conditions and select skilled professionals intended to assume the role of reliable "reviewers" when we will launch WKI into its real environment. This reviewers-selection-process is a key issue to implement in order to help the administrators to detect and filter intentional misinformation or misdirection and ensure the quality of all data published on this collaborative platform.

In another hand, being aware that companies consider collected content as a strategic asset, one reference person per organization is designated to be in charge of the validation of the information filled about his organization. 


\subsection{Integration of a Data-Extraction System to Drag up Information}

We integrate a back-office module to collect qualitative and quantitative data concerning contributors' profiles, their web pages research, the evolution of their consultations and the nature of the information they filled on the WKI. In a second time, the data collected will be processed to build a global diagnostic. The purpose is to build statistical outputs to implement most efficient regional policies toward innovation incentives relying on a qualified raw organization's needs, practices and individuals expectations.

\subsection{Validation Process of the Information}

Each new information created by contributors on WKI passes through a multi-phase validation process which lead to an eventual blending or selection of ideas submitted. In this way, we implement in addition with the reviewers system described above, a voting system for each contribution filled on our WKI to give more details regarding to the success of each contribution. The purpose is to provide another tool to the administrators to help them in their final validation and classification of the contents. In addition, we highlight that a part of this information will be already filtrated by an automatical integrated robot to reduce nuisances and the risk of intrusions.

\subsection{Users Motivating Process}

To motivate users, we intend to offer an incentivizing system by unlocking "premiums functions" all along their evolution toward new status and through an incremental information filling process of their profiles and affiliated organizations. We offer so a plurality of users profiles starting from the simple "contributor" to most knowledgeable expert ones as "reviewers", having then the possibility to qualify others users contributions in order to facilitate the validation work dedicated to the "administrators". This attempts to enhance progressively the pertinence and the quality of the contributions provided by the users. Indeed, the evolution within the WKI community will be motivated by reaching progressively the access to refined data and much more pertinent and personalized information.

\section{Conclusions and Future Work}

In this paper we have presented our principles for designing $\mathrm{WKI}$, an open and collaborative platform dedicated to support innovation and creativity processes. We described its features and technical prerequisites that might answer the difficulties we identified in the previous related works. The objective is to offer an innovative way of processing raw information to broadcast a new perspective and horizons to consider differently innovation processes in enterprises, especially within SMEs. WKI is already in the developing stage. First, we design it to be used initially in Aquitaine region but we expect to export it in others regions or countries to provide then a benchmarking enabling to produce comparative statistics and analysis for all the stakeholders involved within the community. Secondly, the open and scalable dimension of WKI is destined to implement it as a collaborative platform to reduce the existing gap between 
the specifics SMEs needs and the actual traditional answers usually given to them. In addition, by developing the information-crossing potential and the contacts it enables between both academic and entrepreneurial worlds, WKI is expected to reduce the lack of collaboration existing, in France maybe more than anywhere else, between those two environments.

Acknowledgements. Authors would like to acknowledge our partner AGEFA PME for its support concerning the Chair on SMEs 3.0 and the TRANSCREATIVA project which has been co-financed by the "Fonds européen de développement régional" (FEDER) in the frame of the Interreg IV B program, "le programme de coopération territoriale de l'espace Sud-ouest européen" (SUDOE).

\section{References}

1. Boujut J.F., Tiger H.: A socio-technical research method for analyzing and instrumenting the design activity. Journal of Design Research. Vol. 2, Issue 2 (2002)

2. Howe, J.: The Rise of Crowdsourcing. Wired Magazine. Issue 14.06 (2006)

3. Borter, S., Nyffeler, N., Bergeron, L.: Analyse d'une Démarche Transdisciplinaire Favorisant l'Innovation au Sein des PME. In: $10^{\text {ème }}$ Congrès International Francophone en Entrepreneuriat et PME (CIFEPME), Bordeaux (2010)

4. Gallais, M., Bayad, M.: Accompagner Autrement le Dirigeant de PME Vers I'Innovation: Une Exploration du Rapport de Prescription. In: $10^{\text {ème }}$ Congrès International Francophone en Entrepreneuriat et PME (CIFEPME), Bordeaux (Oct. 2010)

5. AFNOR: Management de l'Innovation - Guide de Mise en CEuvre d'une Démarche de Management de l'Innovation. Technical report, FD X 50-271 (4 Décembre 2013)

6. Pascal Morand, P., Manceau., D.: Pour une Nouvelle Vision de I'Innovation. Technical report, ESCP Europe (Avril 2009)

7. Bpifrance: PME 2013, Rapport sur l'Évolution des PME. Technical report, L'Observatoire des PME (2014)

8. Publications Office of the European Union: Horizon 2020 In Brief, The EU Framework Programme for Research and Innovation. Technical report, European Commission (2014)

9. ADE (Aide à la Décision Économique)., LL\&A (Louis Lengrand Associés).: Étude sur l'Évolution des Diagnostics et des Stratégies Régionales d'Innovation Dans les Régions Françaises Dans le Cadre des PO FEDER 2007-2013. Technical report, Rapport Final (Synthèse) (Juillet 2010)

10. Direction Générale de la Compétitivité, de l'Industrie et des Services (DGCIS): L'Innovation Dans les Entreprises Moteurs, Moyens et Enjeux. Technical report, Ministère de l'Économie des Finances et de l'Industrie (Mai 2011)

11. European Commission: Guide to Social Innovation. Technical report, Regional and Urban Policy (February 2013)

12. ARF (Association des Régions de France)., Avise (Ingénierie et Services pour Entreprendre Autrement).: L'Innovation Sociale, Un Levier Pour le Développement Des Territoires - Repères et Bonnes Pratiques pour Développer des Politiques Régionales de Soutien à l'Innovation. Technical report, (Juillet 2012)

13. Organisation de Coopération et de Développement Économiques., Office

Statistique des Communautés Européennes.: Manuel d'Oslo: Principes Directeurs pour le Recueil et l'Interprétation des Données sur l'Innovation - Troisième Edition, la Mesure des Activités Scientifiques et Technologiques. Technical report, OECD (2005) 14. Eurostat: The Community Innovation Survey 2010 (CIS 2010), The Harmonized Survey Questionnaire. Technical report, Final Version (July 9, 2010) 